\title{
EFFECT OF VISCOUS DISSIPATION AND THERMOPORESIS ON THE FLOW OVER AN EXPONENTIALLY STRETCHING SHEET
}

\author{
D. SRINIVASACHARYA* and P. JAGADEESHWAR \\ Department of Mathematics, National Institute of Technology \\ Warangal-506004, Telangana State, INDIA \\ E-mail: dsrinivasacharya@gmail.com
}

\begin{abstract}
This article analyses the influence of viscous dissipation and thermoporesis effects on the viscous fluid flow over a porous sheet stretching exponentially by applying convective boundary condition. The numerical solutions to the governing equations are evaluated using a local similarity and non-similarity approach along with a successive linearisation procedure and Chebyshev collocation method. The influence of the pertinent parameters on the physical quantities are displayed through graphs.
\end{abstract}

Key words: thermophoresis, viscous dissipation, Eckert number.

\section{Introduction}

The study of flow, heat and mass transfer over stretching surfaces is one of the important research area due to its significant use in various chemical, polymer industries and other engineering disciplines. Applications include wire drawing, crystal growth, filaments spinning, paper production, glass fiber, food processing, continuous casting etc. Sakiadis $[1,2]$ was the first to study the flow due to a stretching sheet. Since then several researchers have analyzed this flow problem including the heat and mass transfer analysis under several physical situations, e.g., over a stretching/shrinking sheet with convective boundary condition. They concluded that the convective boundary conditions result in a temperature slip at the wall.

Thermophoresis is a mechanism in which small particles migrate in the direction of decreasing thermal gradient. It is quite significant in radioactive particle deposition in nuclear reactor safety simulations, aerosol particle sampling, deposition of silicon thin films, etc. Goldsmith and May [3] were the first to estimate the thermophoretic velocity in a one-dimensional flow. Uddin et al. [4] studied the thermophoresis and magnetic field effect over a linearly stretching sheet. Shehzad et al. [5] analyzed the effects of the magnetic field, radiation, thermoporesis and Joule heating effects on the flow of Jeffrey fluid over a linearly stretched surface. Reddy [6] investigated the impact of thermophoresis and variable thermal conductivity on MHD viscous fluid flow over an inclined surface. Sandeep and Sulochana [7] studied the nanofluid flow over an exponentially stretching porous sheet immersed in a porous medium in the presence of thermophoresis, radiation and magnetic field.

The process of transforming the energy taken from the motion of the fluid by viscosity into internal energy, which is partially irreversible, is referred to as viscous dissipation. Gehbart [8] considered the significance of viscous dissipation on natural convection. Wong et al. [9] investigated viscous dissipation effect on the steady viscous fluid flow over an exponentially stretching/shrinking permeable sheet. Das [10] investigated the effect of chemical reaction and viscous dissipation on MHD mixed convective heat and mass transfer flow of a second grade fluid past a semi-infinite stretching sheet in the presence of thermal diffusion and thermal radiation. Megahed [11] studied the heat transfer characteristics in the presence of viscous dissipation and velocity slip of a viscous Casson thin film flow over an unsteady stretching sheet. Adeniyan

\footnotetext{
* To whom correspondence should be addressed
} 
and Adigun [12] reported that increasing the values of the Eckert number and magnetic parameter, the thermal boundary layer thickness is increasing.

A generally accepted boundary condition on the solid surface is no-slip condition. However, Navier [13] suggested that fluid slips at the solid boundary and slip velocity depend linearly on the shear stress. The fluid slippage phenomenon at the solid boundary appears in numerous applications, for example, in nanochannels or microchannels and the cleaning of simulated heart valves, internal cavities. On the other hand, a novel technique for the heating process by providing the heat with finite capacity to the convecting fluid through the bounding surface has attracted numerous researchers. This type of thermal boundary condition, called convective boundary condition, results in the rate of exchange of heat across the boundary being proportional to the difference in local temperature with the ambient conditions [14]. Due to the realistic nature of the convective thermal condition, the investigation of heat transfer with this condition has great significance in mechanical and designing fields, for example, heat exchangers, atomic plants, gas turbines, and so forth. Gideon and Abah [15] studied plane stagnation double-diffusive MHD convective flow with convective boundary condition in porous media. Mustafaa et al. [16] reported the impact of convective boundary condition on the heat transfer characteristics past an exponentially stretching sheet in a nanofluid considering the thermophoresis and Brownian motion effects. Hayat et al. [17] studied the effects of convective heat and mass transfer in the flow of Eyring-Powell fluid past an inclined exponential stretching surface. Rahman et al. [18] investigated the steady boundary layer flow and heat transfer characteristics of a nanofluid past an exponentially shrinking surface with convective boundary condition. Mabood et al. [19] investigated the stagnation point flow and heat transfer over an exponential stretching sheet. Khan et al. [20] studied the boundary layer flow of a nanofluid past a bi-directional exponentially stretching sheet with convective boundary condition. Recently, Srinivasacharya and Jagadeeshwar [21] reported that the increase in the Biot number increases the rate of heat transfer from the sheet to the fluid.

Therefore, motivated by the aforesaid investigations, here we made an attempt to analyze the boundary layer flow considering the effects of thermophoresis and viscous dissipation over an exponential stretching surface subjected to velocity slip and suction or injection.

\section{Mathematical formulation}

Consider a stretching sheet in a laminar slip flow of a viscous incompressible fluid with temperature $T_{\infty}$ and concentration $C_{\infty}$. The Cartesian framework is selected by taking the positive $\tilde{x}$-axis along the sheet and the $\tilde{y}$-axis orthogonal to the sheet. The stretching velocity of the sheet is assumed as $U_{*}(\tilde{x})=U_{0} e^{\tilde{x} / L}$ where $\tilde{x}$ is the distance from the slit. Assume that the sheet is either cooled or heated convectively through a fluid with temperature $T_{f}$ and which induces a heat transfer coefficient $h_{f}$, where $h_{f}=h \sqrt{U_{0} / 2 L} e^{\tilde{x} / 2 L}$. $\left(\tilde{u}_{x}, \tilde{u}_{y}\right)$ is the velocity vector, $\tilde{C}$ is the concentration and $\tilde{T}$ is the temperature. The suction/injection velocity of the fluid through the sheet is $V_{*}(\tilde{x})=V_{0} e^{\tilde{x} / 2 L}$, where $V_{0}$ is the strength of suction/injection. Further, the slip velocity of the fluid is assumed to be $N(\tilde{x})=N_{0} e^{-\tilde{x} / 2 L}$, where $N_{0}$ is the velocity slip factor. Hence, the following are the equations which govern the present flow

$$
\begin{aligned}
& \frac{\partial \tilde{u}_{x}}{\partial \tilde{x}}+\frac{\partial \tilde{u}_{y}}{\partial \tilde{y}}=0, \\
& \tilde{u}_{x} \frac{\partial \tilde{u}_{x}}{\partial \tilde{x}}+\tilde{u}_{y} \frac{\partial \tilde{u}_{x}}{\partial \tilde{y}}=v \frac{\partial^{2} \tilde{u}_{x}}{\partial \tilde{y}^{2}}, \\
& \tilde{u}_{x} \frac{\partial \tilde{T}}{\partial \tilde{x}}+\tilde{u}_{y} \frac{\partial \tilde{T}}{\partial \tilde{y}}=\alpha \frac{\partial^{2} \tilde{T}}{\partial \tilde{y}^{2}}+\frac{\mu}{\rho c_{p}}\left(\frac{\partial \tilde{u}_{x}}{\partial \tilde{y}}\right)^{2},
\end{aligned}
$$




$$
\tilde{u}_{x} \frac{\partial \tilde{C}}{\partial \tilde{x}}+\tilde{u}_{y} \frac{\partial \tilde{C}}{\partial \tilde{y}}=D \frac{\partial^{2} \tilde{C}}{\partial \tilde{y}^{2}}-\frac{\partial}{\partial y}\left[V_{T}\left(\tilde{C}-C_{\infty}\right)\right]
$$

where $\rho$ is density, $D$ is the mass diffusivity of the medium, $\alpha$ is the thermal diffusivity, $v$ is the kinematic viscosity of the fluid, $c_{p}$ is the specific heat capacity at the constant pressure and $V_{T}$ is the thermophoretic velocity.

The term $V_{T}$ in Eq.(2.4) can be written as [22]

$$
V_{T}=-\frac{v k_{t}}{T_{r}} \frac{\partial \tilde{T}}{\partial \tilde{y}}
$$

where $T_{r}$ is the reference temperature and $k_{t}$ is the thermophoretic coefficient.

The conditions on the surface of the stretching sheet are

$$
\begin{aligned}
& \tilde{u}_{x}=U_{*}+N v \frac{\partial \tilde{u}_{x}}{\partial \tilde{y}}, \quad \tilde{u}_{y}=-V_{*}(\tilde{x}), \quad-\kappa \frac{\partial \tilde{T}}{\partial \tilde{y}}=h_{f}\left(T_{f}-\tilde{T}\right), \quad \tilde{C}=C_{w} \quad \text { at } \quad \tilde{y}=0, \\
& \tilde{u}_{x} \rightarrow 0, \quad \tilde{T} \rightarrow T_{\infty}, \quad \tilde{C} \rightarrow C_{\infty} \quad \text { as } \quad \tilde{y} \rightarrow \infty .
\end{aligned}
$$

Introducing the stream functions through $\tilde{u}_{x}=-\frac{\partial \psi}{\partial \tilde{y}}$ and $\tilde{u}_{y}=\frac{\partial \psi}{\partial \tilde{x}}$ and then the following dimensionless variables

$$
\begin{aligned}
& x=\frac{\tilde{x}}{L}, \quad y=\tilde{y} \sqrt{\frac{U_{0}}{2 v L} e^{\frac{\tilde{x}}{2 L}},} \quad \psi=\sqrt{2 v L U_{0}} e^{\frac{\tilde{x}}{2 L}} F(x, y), \\
& \tilde{T}=T_{\infty}+\left(T_{f}-T_{\infty}\right) T(x, y), \quad \tilde{C}=C_{\infty}+\left(C_{w}-C_{\infty}\right) C(x, y),
\end{aligned}
$$

into Eqs (2.1)-(2.4), we obtain

$$
\begin{aligned}
& F^{\prime \prime \prime}+F F^{\prime \prime}-2 F^{2}+2\left(F^{\prime \prime} \frac{\partial F}{\partial x}-F^{\prime} \frac{\partial F^{\prime}}{\partial x}\right)=0 \\
& \frac{1}{\operatorname{Pr}} T^{\prime \prime}+F T^{\prime}+\mathrm{Ec} e^{2 x} F^{\prime \prime 2}+2\left(T^{\prime} \frac{\partial F}{\partial x}-F^{\prime} \frac{\partial T}{\partial x}\right)=0 \\
& \frac{1}{\mathrm{Sc}} C^{\prime \prime}+F C^{\prime}-\tau\left(T^{\prime} C^{\prime}+C T^{\prime \prime}\right)+2\left(C^{\prime} \frac{\partial F}{\partial x}-F^{\prime} \frac{\partial C}{\partial x}\right)=0
\end{aligned}
$$

where the prime indicates derivative with respect to $y, S=V_{0} \sqrt{2 L / v U 0}$ is the suction or blowing parameter according to $S>0$ or $S<0$, respectively, $\mathrm{Ec}=U_{0}{ }^{2} / c_{p}\left(T_{f}-T_{\infty}\right)$ is the Eckert number, $\operatorname{Pr}=v / \alpha$ is the Prandtl number, $\mathrm{Sc}=v / D$ is the Schmidt number, $\mathrm{Bi}=h \sqrt{v} / \kappa$ is the Biot number, $\lambda=N_{0} \sqrt{\nu U_{0} / 2 L}$ is the velocity slip parameter and $\tau=-k_{t}\left(T_{w}-T_{\infty}\right) / T_{r}$ is the thermophoretic parameter (The surface is cold for $\tau$ $>0$ and hot for $\tau<0[23,24])$. 
The transformed boundary conditions are

$$
\begin{aligned}
& F(x, 0)+2 \frac{\partial F}{\partial x}(x, 0)=S, \quad F^{\prime}(x, 0)=1+\lambda F^{\prime \prime}(x, 0), \\
& T^{\prime}(x, 0)=-\operatorname{Bi}(1-T(x, 0)), \quad C(x, 0)=1, \\
& F^{\prime}(x, y) \rightarrow 0, \quad T(x, y) \rightarrow 0, \quad C(x, y) \rightarrow 0 \quad \text { as } \quad y \rightarrow \infty .
\end{aligned}
$$

The non-dimensional skin friction $C_{f}=2 \tau_{\omega} / \rho U^{2}$, the local Nusselt number $\mathrm{Nu}_{\tilde{x}}=\tilde{x} q_{w} / k\left(T_{f}-T_{\infty}\right)$, and the local Sherwood number $S h_{\tilde{x}}=\tilde{x} q_{m} / k\left(C_{w}-C_{\infty}\right)$, are given by

$$
\sqrt{\frac{L}{2 x}} \sqrt{\operatorname{Re}_{x}} C_{f}=F^{\prime \prime}(x, 0), \quad \sqrt{\frac{2}{L x}} \frac{\mathrm{Nu}_{x}}{\sqrt{\mathrm{Re}_{x}}}=-T^{\prime}(x, 0) \quad \text { and } \quad \sqrt{\frac{2}{L x}} \frac{\mathrm{Sh}_{x}}{\sqrt{\mathrm{Re}_{x}}}=-C^{\prime}(x, 0)
$$

where $\operatorname{Re}_{x}=\frac{x U(x)}{v}$ is the local Reynolds number.

\section{Numerical solution}

The numerical solutions to Eqs (2.8) to (2.10) together with Eqs (2.11) are evaluated using a local similarity and non-similarity method [25], [26], [27], successive linearisation and then the pseudo spectral method [28], [29].

\subsection{Local non-similarity method}

The initial approximate solution can be obtained from the local similarity equations for a particular case $x<<1$ by suppressing the terms $x(\partial / \partial x)$. As there are no terms accompanied with $x(\partial / \partial x)$ in Eqs (2.8)(2.10), there is no change in the governing equations and boundary conditions.

In the second step, introduce $G=\partial F / \partial x, H=\partial T / \partial x$ and $K=\partial C / \partial x$ to get back the suppressed terms in the first step.

The subsequent solutions for $f_{i}(\eta), \theta_{i}(\eta)$ and $\phi_{i}(\eta)(i \geq 1)$ are obtained by recursively solving the following linearized system of ordinary differential equations

$$
\begin{aligned}
& F^{\prime \prime \prime}+F F^{\prime \prime}-2 F^{2}+2\left(F^{\prime \prime} G-F^{\prime} G^{\prime}\right)=0, \\
& \frac{1}{\operatorname{Pr}} T^{\prime \prime}+F T^{\prime}+\operatorname{Ec} e^{2 x} F^{\prime \prime 2}+2\left(T^{\prime} G-F^{\prime} H\right)=0, \\
& \frac{1}{\mathrm{Sc}} C^{\prime \prime}+F C^{\prime}-\tau\left(T^{\prime} C^{\prime}+C T^{\prime \prime}\right)+2\left(C^{\prime} G-F^{\prime} K\right)=0 .
\end{aligned}
$$

The associated boundary conditions are 


$$
\begin{aligned}
& F(x, 0)+2 G(x, 0)=S, \quad F^{\prime}(x, 0)=1+\lambda F^{\prime \prime}(x, 0), \\
& T^{\prime}(x, 0)=-B i(1-T(x, 0)), \quad C(x, 0)=1, \\
& F^{\prime}(x, \infty) \rightarrow 0, \quad T(x, \infty) \rightarrow 0, \quad C(x, \infty) \rightarrow 0 .
\end{aligned}
$$

In the third step, differentiate Eqs (3.1)-(3.3) with respect to $x$ and neglect terms accompanied with $\partial G / \partial x, \partial H / \partial x$ and $\partial K / \partial x$, then we get

$$
\begin{aligned}
& G^{\prime \prime \prime}+F G^{\prime \prime}+G F^{\prime \prime}-4 F^{\prime} G^{\prime}+2\left(G^{\prime \prime} G-G^{2}\right)=0, \\
& \frac{1}{\operatorname{Pr}} H^{\prime \prime}+\left(F H^{\prime}+G T^{\prime}\right)+2 \mathrm{Ec} e^{2 x}\left(F^{\prime \prime 2}+F^{\prime \prime} G^{\prime \prime}\right)+2\left(H^{\prime} G-G^{\prime} H\right)=0, \\
& \frac{1}{\mathrm{Sc}} K^{\prime \prime}+\left(F K^{\prime}+G C^{\prime}\right)-\tau\left(H^{\prime} C^{\prime}+T^{\prime} K^{\prime}+K T^{\prime \prime}+C H^{\prime \prime}\right)+2\left(K^{\prime} G-G^{\prime} K\right)=0 .
\end{aligned}
$$

The associated boundary conditions are

$$
\begin{aligned}
& G(x, 0)=0, \quad G^{\prime}(x, 0)=\lambda G^{\prime \prime}(x, 0), \quad H^{\prime}(x, 0)=B i H(x, 0), \quad K(x, 0)=0, \\
& G^{\prime}(x, \infty) \rightarrow 0, \quad H(x, \infty) \rightarrow 0, \quad K(x, \infty) \rightarrow 0 .
\end{aligned}
$$

\subsection{Successive linearization method}

Let $\Gamma(y)=[F, T, C, G, H, K]$ and assume that

$$
\Gamma(y)=\Gamma_{i}(y)+\sum_{r=0}^{i-1} \Gamma_{r}(y)
$$

where $\Gamma_{i}(y)(i=1,2,3, \ldots)$ are unknown functions that are determined by recursively evaluating the linearised version of Eqs (3.1) to (3.8) after introducing Eq.(3.9) into them and $\Gamma_{r}(y)(r \geq 1)$ are known functions determined from previous iterations

$$
\begin{aligned}
& F_{i}{ }^{\prime \prime}+\chi_{11, i-1} F_{i}{ }^{\prime}+\chi_{12, i-1} F_{i}{ }^{\prime}+\chi_{13, i-1} F_{i}+\chi_{14, i-1} G_{i}{ }^{\prime}+\chi_{15, i-1} G_{i}=\zeta_{1, i-1}, \\
& \chi_{21, i-1} F_{i}{ }^{\prime \prime}+\chi_{22, i-1} F_{i}{ }^{\prime}+\chi_{23, i-1} F_{i}+\frac{1}{\operatorname{Pr}} T_{i}{ }^{\prime \prime}+\chi_{24, i-1} T_{i}{ }^{\prime}+\chi_{25, i-1} G_{i}+\chi_{26, i-1} H_{i}=\zeta_{2, i-1}, \\
& \chi_{31, i-1} F_{i}{ }^{\prime}+\chi_{32, i-1} F_{i}+\chi_{33, i-1} T_{i}{ }^{\prime \prime}+\chi_{34, i-1} T_{i}{ }^{\prime}+\frac{1}{\mathrm{Sc}} C_{i}{ }^{\prime \prime}+ \\
& +\chi_{35, i-1} C_{i}{ }^{\prime}+\chi_{36, i-1} C_{i}+\chi_{37, i-1} G_{i}+\chi_{38, i-1} K_{i}=\zeta_{3, i-1}, \\
& \chi_{41, i-1} F_{i}{ }^{\prime \prime}+\chi_{42, i-1} F_{i}{ }^{\prime}+\chi_{43, i-1} F_{i}+G_{i}{ }^{\prime \prime}+\chi_{44, i-1} G_{i}{ }^{\prime}+\chi_{45, i-1} G_{i}{ }^{\prime}+\chi_{46, i-1} G_{i}=\zeta_{4, i-1}, \\
& \chi_{51, i-1} F_{i}{ }^{\prime \prime}+\chi_{52, i-1} F_{i}+\chi_{53, i-1} T_{i}{ }^{\prime}+\chi_{54, i-1} G_{i}{ }^{\prime \prime}+\chi_{55, i-1} G_{i}{ }^{\prime}+ \\
& +\chi_{56, i-1} G_{i}+\frac{1}{\operatorname{Pr}} H_{i}{ }^{\prime}+\chi_{57, i-1} H_{i}{ }^{\prime}+\chi_{58, i-1} H_{i}=\zeta_{5, i-1},
\end{aligned}
$$




$$
\begin{aligned}
& \chi_{61, i-1} F_{i}+\chi_{62, i-1} T_{i}{ }^{\prime}+\chi_{63, i-1} T_{i}{ }^{\prime}+\chi_{64, i-1} C_{i}{ }^{\prime}+\chi_{65, i-1} C_{i}+ \\
& +\chi_{66, i-1} G_{i}{ }^{\prime}+\chi_{67, i-1} G_{i}+\chi_{68, i-1} H_{i}{ }^{\prime}+\chi_{69, i-1} H_{i}{ }^{\prime}+\frac{1}{\mathrm{Sc}} K_{i}{ }^{\prime}+ \\
& +\chi_{610, i-1} K_{i}{ }^{\prime}+\chi_{611, i-1} K_{i}=\zeta_{6, i-1}
\end{aligned}
$$

where the coefficients $\chi_{l k, n-1}$ and $\zeta_{k, i-1},(l=1,2,3,4,5,6, k=1,2,3, \ldots, 11)$ are in terms of the approximations $F_{i}, T_{i}$ and $C_{i},(i=1,2,3, \ldots, n-1)$ and their derivatives.

\subsection{Chebyshev collocation method}

The linearized equations obtained in section (3.2) are solved using the Chebyshev collocation procedure [29]. In view of numerical computations, the region $[0, \infty)$ is truncated to $[0, L]$ for large $L$. In order to apply the Chebyshev collocation procedure, the interval $[0, L]$ is converted to $[-1,1]$ by the mapping

$$
\tilde{\tau}=\frac{2 y}{L}-1, \quad-1 \leq \tilde{\tau} \leq 1 .
$$

The unknown functions $\Gamma_{i}(y) \quad(i=1,2,3, \ldots)$ and their derivatives can be expressed in terms of the Chebyshev polynomials $\mathrm{Y}_{k}(\tilde{\tau})=\cos \left(k \cos ^{-1}(\tilde{\tau})\right)$ at Gauss-Lobatto collocation points $\tilde{\tau}_{m}=\cos \frac{\pi m}{\mathfrak{K}}, m=0,1,2, \ldots, \mathfrak{K}$ as

$$
\Gamma_{i}(\tilde{\tau})=\sum_{k=0}^{N} \Gamma_{i}\left(\tilde{\tau}_{k}\right) \mathrm{Y}_{k}\left(\tilde{\tau}_{m}\right) \quad \text { and } \quad \frac{d^{a} \Gamma_{r}}{d y^{a}}=\sum_{k=0}^{N} \boldsymbol{D}_{k \boldsymbol{m}}^{\boldsymbol{a}} \Gamma_{i}\left(\tilde{\tau}_{k}\right)
$$

where $\mathbf{D}=\frac{2}{L} \mathcal{D}$ with $\mathcal{D}$ is the Chebyshev derivative matrix and $\boldsymbol{a}$ is the order of the derivative. Substitution of Eq.(3.16) in Eqs (3.10) to (3.15) gives

$$
\mathbb{A}_{i-1} \mathbb{X}_{i}=\mathbb{R}_{i-1}
$$

where $\mathbb{A}_{i-1}$ is a $6^{\text {th }}$ order square matrix with elements as $(\mathfrak{K}+1)^{\text {th }}$ order square matrices in terms of the coefficients $\chi_{i j}$ 's. $\mathbb{X}_{i}$ and $\mathbb{R}_{i-1}$ are $6^{\text {th }}$ order column vectors with $(\mathfrak{K}+1) \times 1$ column vectors as elements in terms of $\Gamma_{i}\left(\tau_{k}\right)$ and $\chi_{s, i-1}$.

Hence, the solution can be obtained by solving the matrix system (3.18), after implementing the boundary conditions.

\section{Results and discussions}

In order to analyze the influence of pertinent parameters, the numerical calculations are carried out by taking $\operatorname{Pr}=1.0, \mathrm{Sc}=0.22, N=100, L=20, S=0.5, \mathrm{Bi}=1.0, \lambda=0.5, \tau=0.3, \mathrm{Ec}=0.2$ and $x=0.2$ unless otherwise mentioned.

The behavior of velocity in the presence of velocity slip at the boundary for exponentially stretching sheet is presented in Fig.1a. Due to the slipperiness velocity decreased. Figure 1b represents the variation of velocity profile in the presence of suction/injection parameter $S$. It is observed that velocity is decreasing by raising the value of $S$. While, a reverse trend is noticed for injection $(S<0)$. 


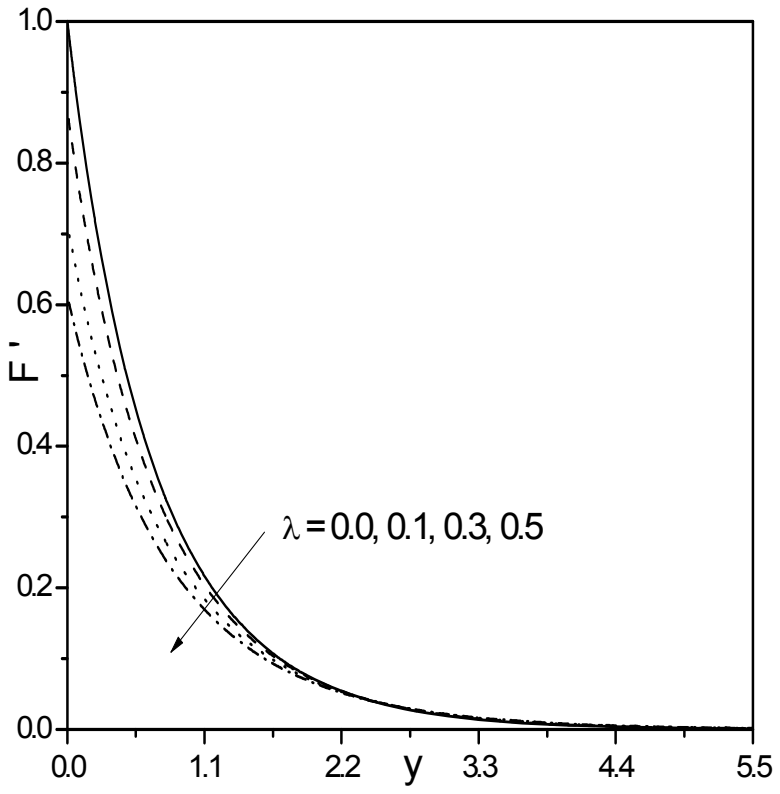

(a)

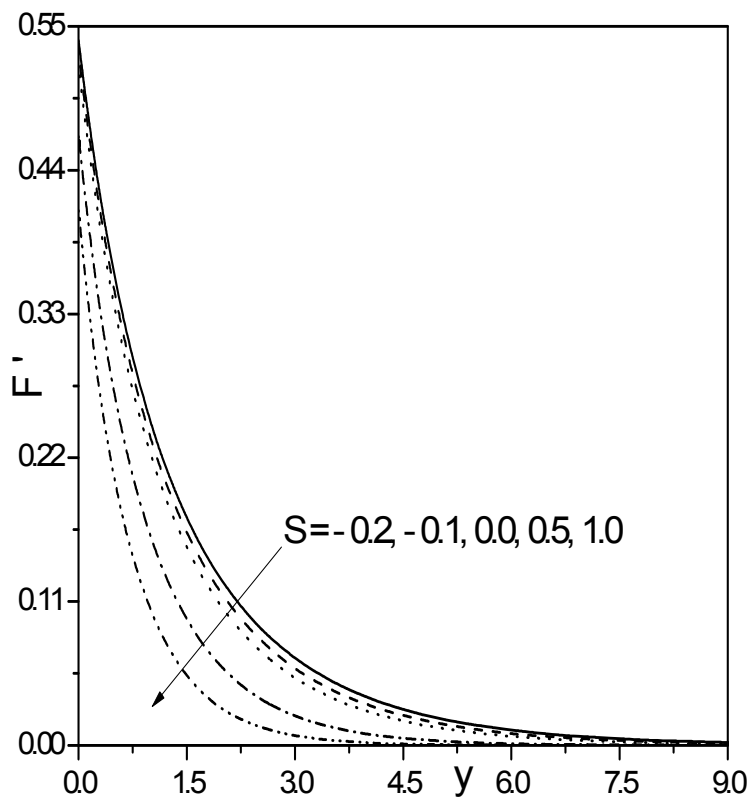

(b)

Fig.1. Effect of (a) $\lambda$ and (b) $S$ on $F^{\prime}$.

The variation of the skin-friction coefficient $F^{\prime \prime}(x, 0)$ against the non-similar variable $x$ for distinct values of slip and suction/injection parameters is presented in Figs $2 \mathrm{a}$ and $2 \mathrm{~b}$. It is obvious from these figures that the skin-friction coefficient is increased with an increase in the values of slip and decreased with a rise in suction parameter. Further, it is identified that the non-similar variable has no impact on the skin-friction coefficient.

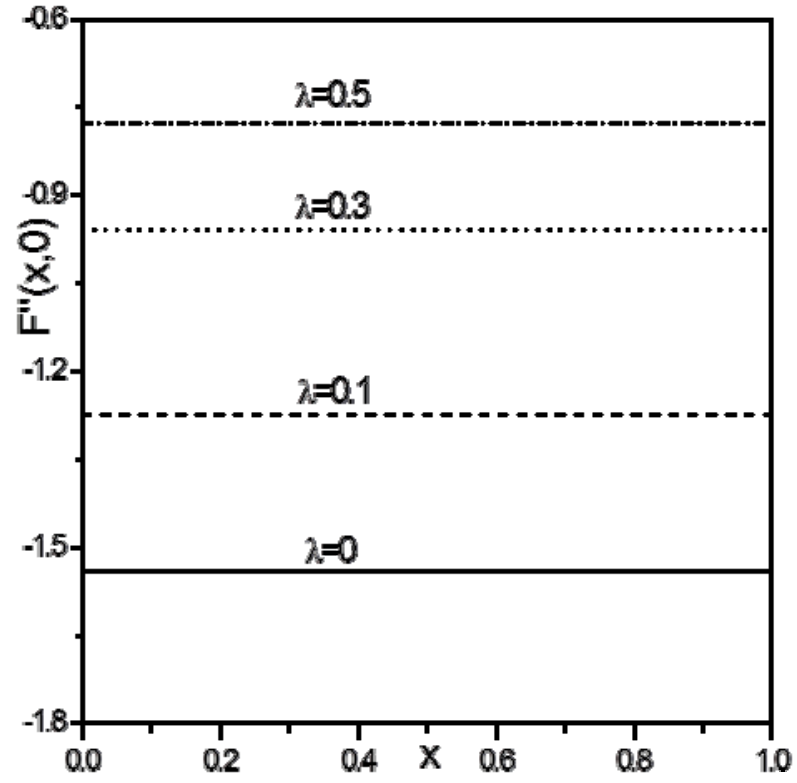

(a)

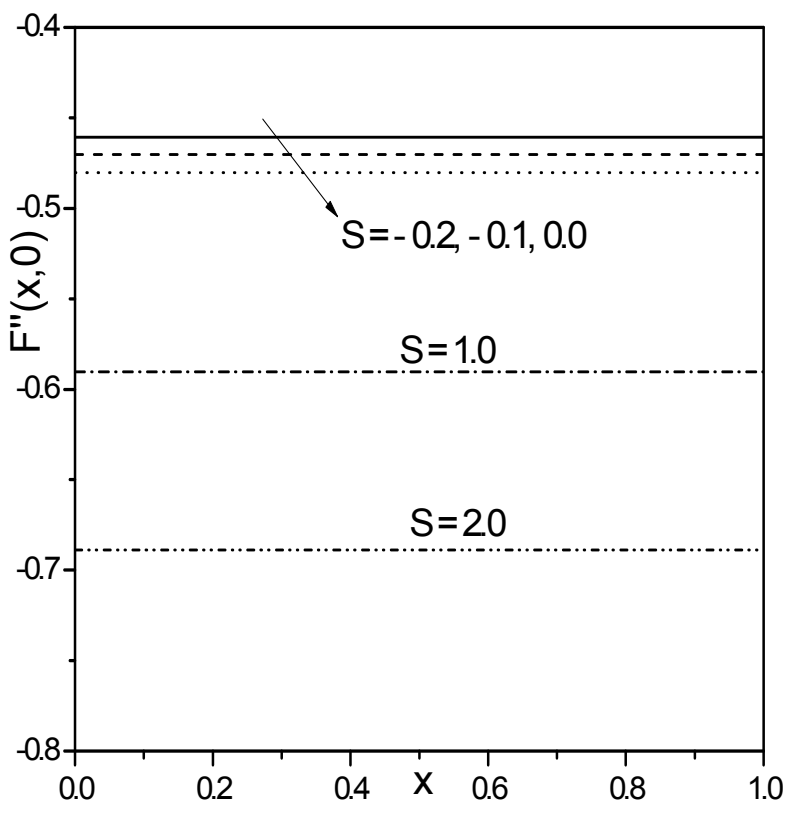

(b)

Fig. 2 Effect of (a) $\lambda$ and (b) $S$ on $F^{\prime \prime}(x, 0)$. 
Figures 3a-3d exhibit the behaviour of temperature profile for different values of Ec, $\mathrm{Bi}, \lambda$ and $S$, respectively. It is evident from Fig.3a that temperature is increasing with an increase in the value of Ec. Figure $3 \mathrm{~b}$ shows that temperature enhanced raising the value of $\mathrm{Bi}$. We see that temperature within the boundary layer increases with an increase in the Biot number. For $\mathrm{Bi} \rightarrow \infty$, Eqs (2.11) implies $T(0) \rightarrow 1$ which is clearly shown in Fig.3b for larger values of Bi. From Fig.3c, it is seen that temperature profile increases with an increase in the values of slip parameter $\lambda$. On the other hand, Fig. $3 d$ shows that temperature of the fluid is decreasing with an increase in the value of the suction parameter and an opposite trend is seen for injection.

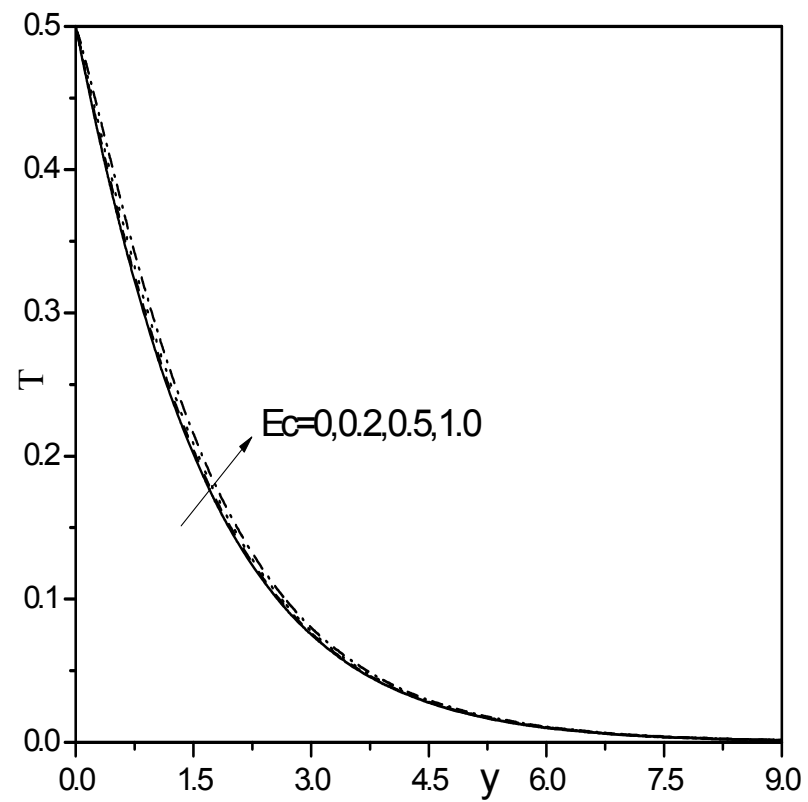

(a)

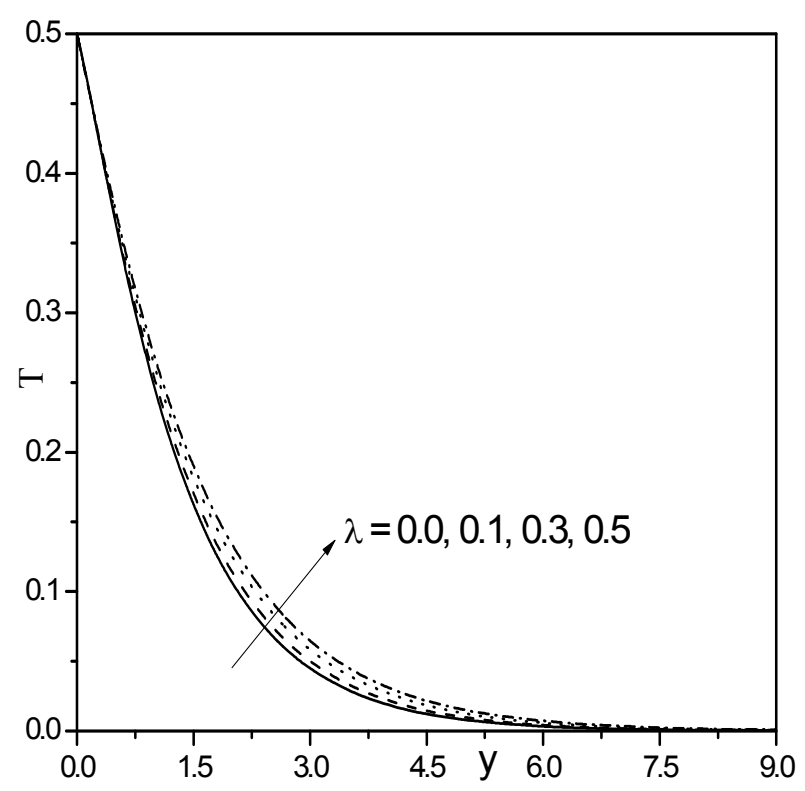

(c)

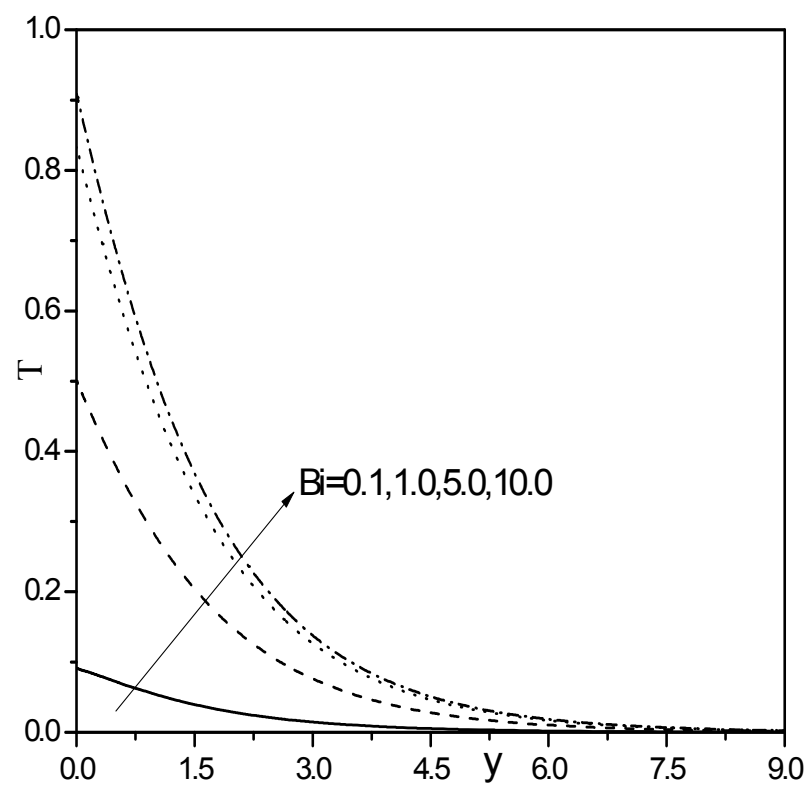

(b)

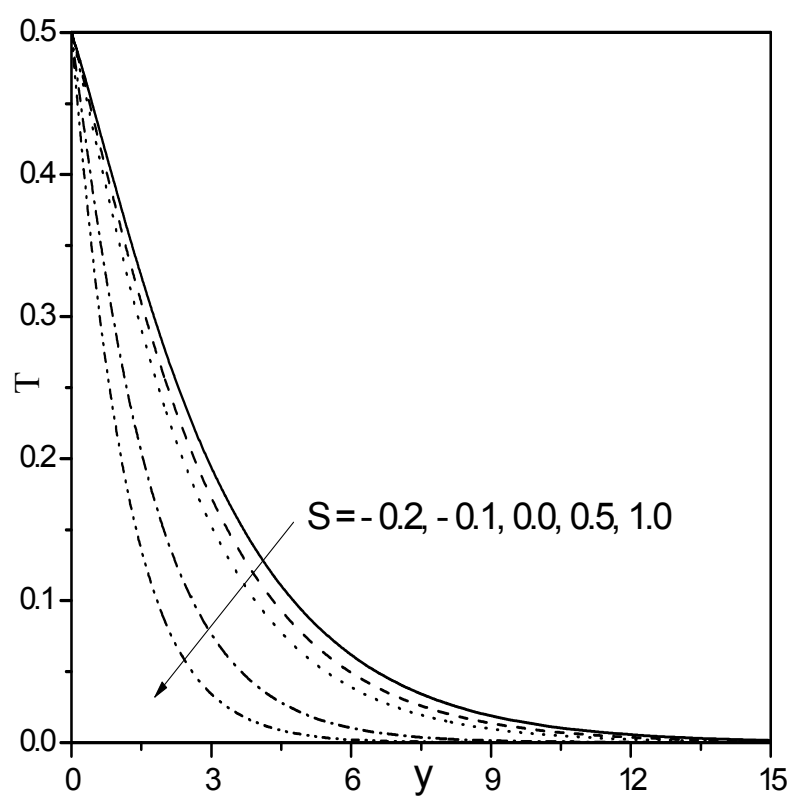

(d)

Fig.3. Effect of (a) Ec, (b) Bi, (c) $\lambda$ and (d) $S$ on $T$. 
The effect of the thermophoretic parameter $\tau$, Biot number Bi, slip parameter $\lambda$ and suction/injection parameter $S$ on concentration profile is presented in Figs 4a-4d. Increase in the value of $\tau$ decreases the concentration as shown in Fig.4a. This is due to the fact that fluid particles move away from cool surroundings with an increase in the thermophoretic parameter. Figure $4 \mathrm{~b}$ shows the influence of convection on concentration. It is known that a rise in convection at the stretching sheet results in lowering thermal penetration and hence decreases the concentration boundary layer thickness. Therefore, mass transfer at the sheet increases with an increase in the value of Bi. Further, as the value of the slip parameter increases, concentration of the fluid increases as shown in Fig.4c. Due to which, mass transfer at the sheet decreases. On the other hand, concentration decreases with increase in the value of suction and increases with increase in the value of injection as shown in Fig.4d.

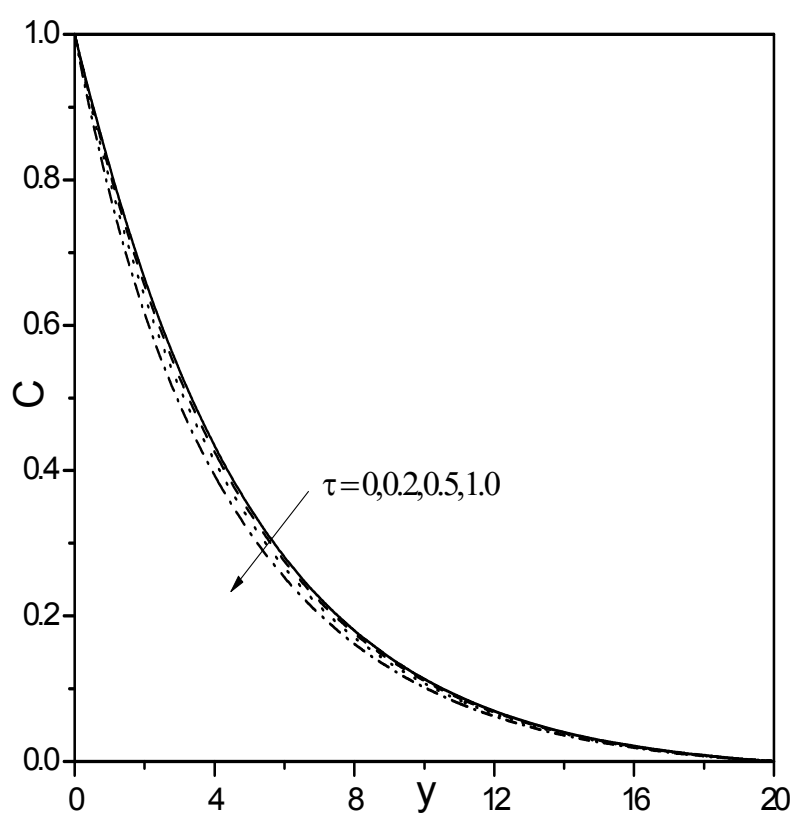

(a)

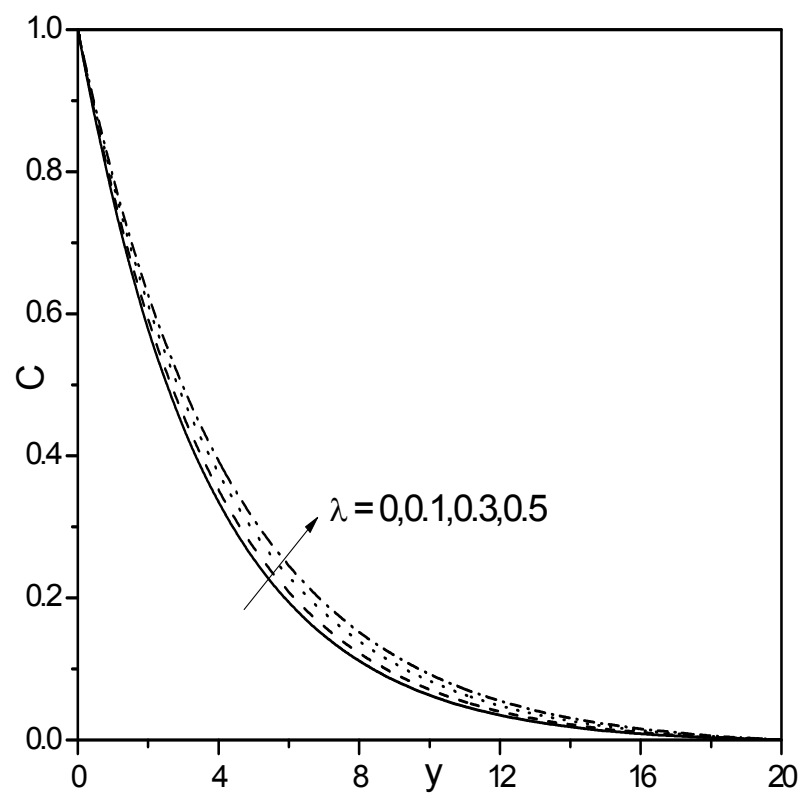

(c)

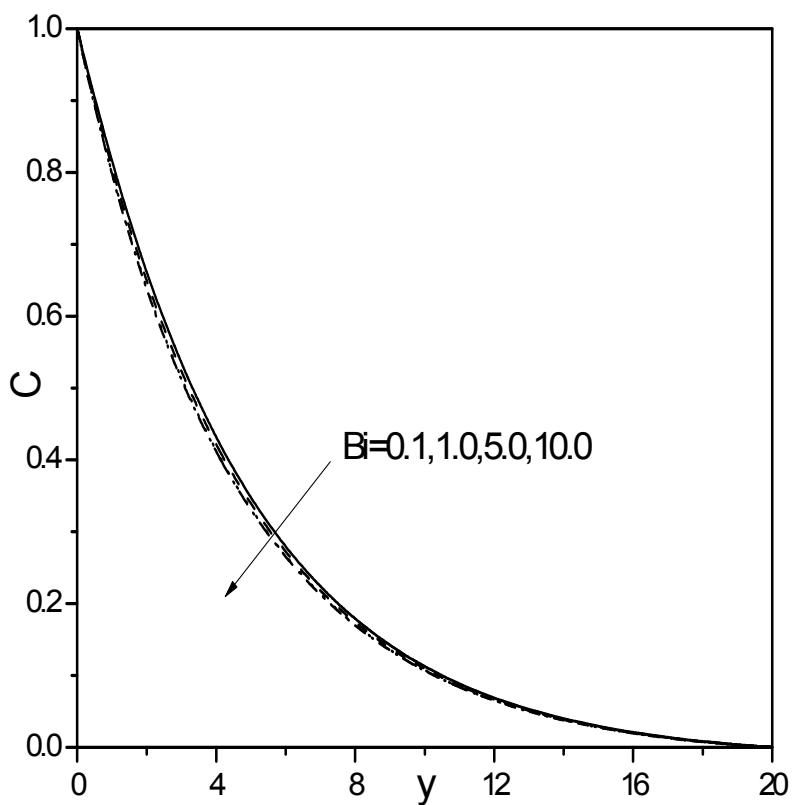

(b)

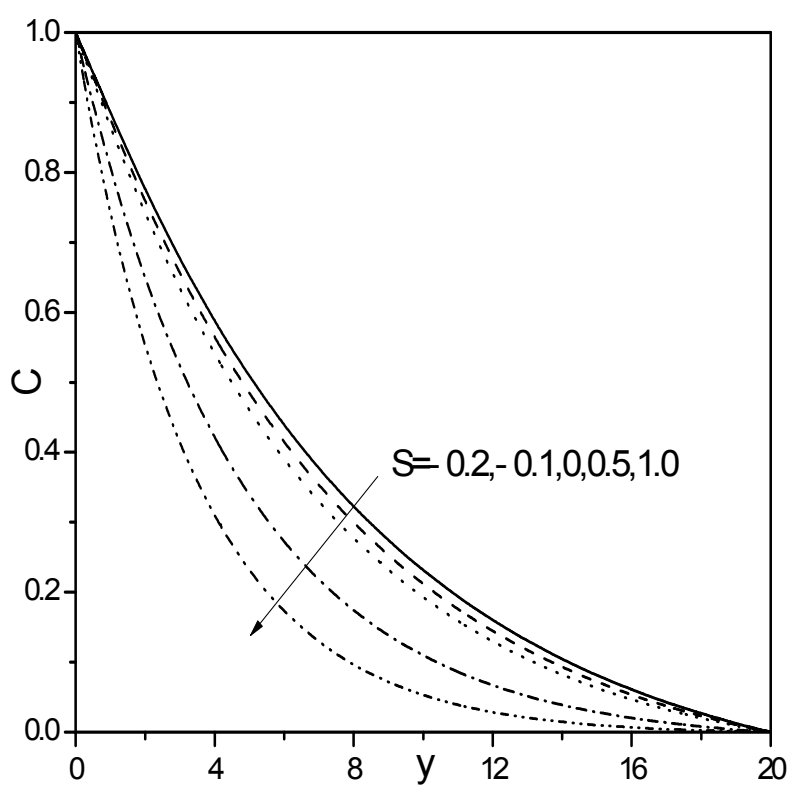

(d)

Fig.4. Effect of (a) $\tau$, (b) Bi, (c) $\lambda$ and (d) $S$ on $C$. 
The fluctuation of the heat transfer coefficient for different values of the Eckert number, Biot number, slip and suction/ijnection parameters against non-similar variable $x$ is presented through Figs 5a-5d. The influence of the Eckert number on the Nusselt number is depicted in Fig.5a. It is evident from the figure that heat transfer from the sheet to the fluid is decreasing with an increase in the value of Ec. In the absence of the Eckert number $(\mathrm{Ec}=0)$, there no effect of the non-similar variable $x$ on the heat transfer coefficient. As the value of Ec increases, heat transfer from the sheet to the fluid increases and as $x \rightarrow 1$ and for higher values of the Eckert number heat absorption takes place. Increasing values of the Biot number the heat transfer coefficient is enhanced predominantly on the surface due to the strong convection as shown in Fig. 5 b. Figure $5 \mathrm{c}$ shows that the rate of heat transfer enhanced with an increase in the slipperiness. But, it is noticed that in the absence of slipperiness and for small values of slipperiness heat absorption is taking place far away from the boundary. While the heat transfer coefficient is increasing with an increase in the value of $S$ as depicted in Fig.5d.

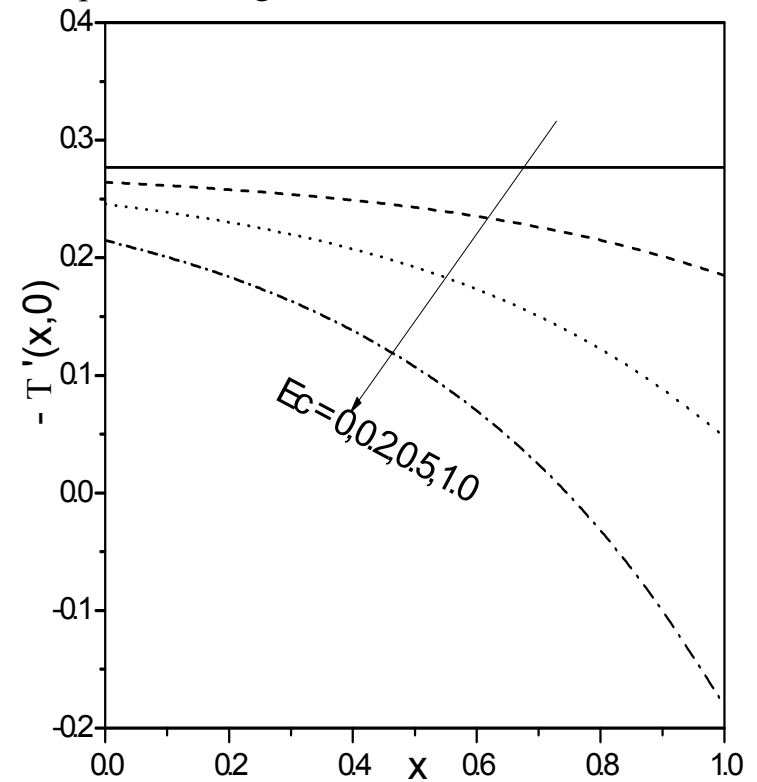

(a)

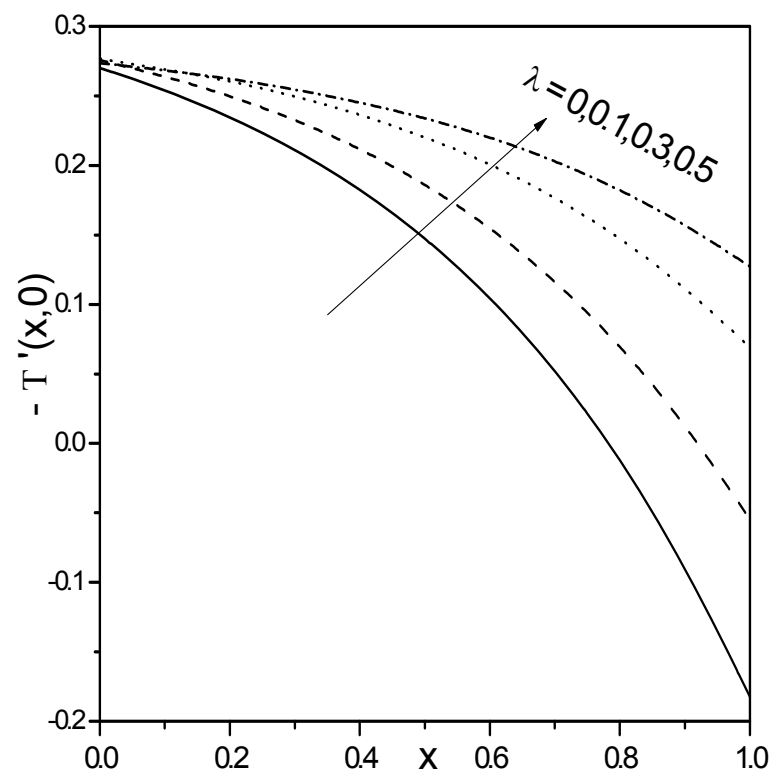

(c)

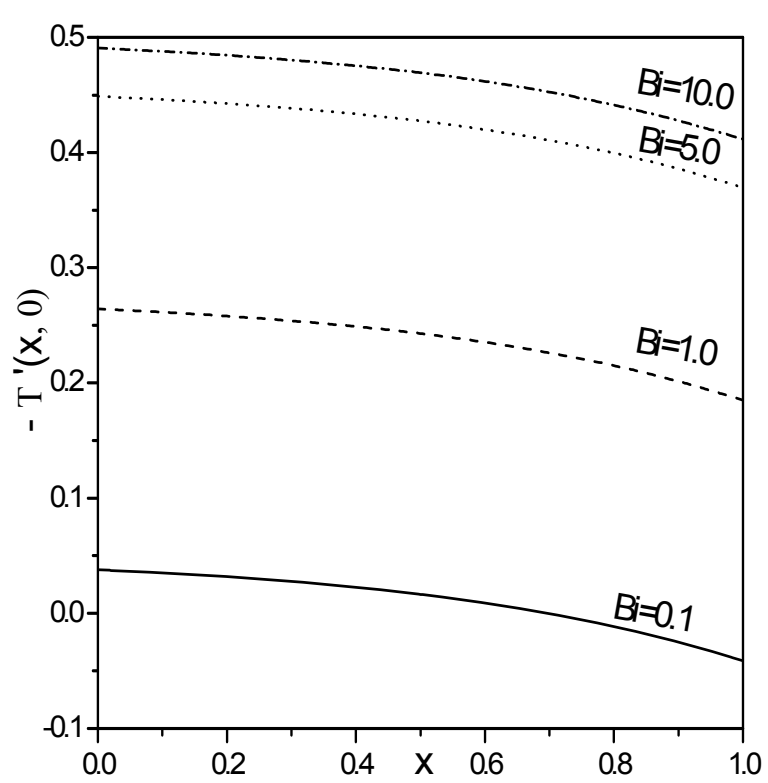

(b)

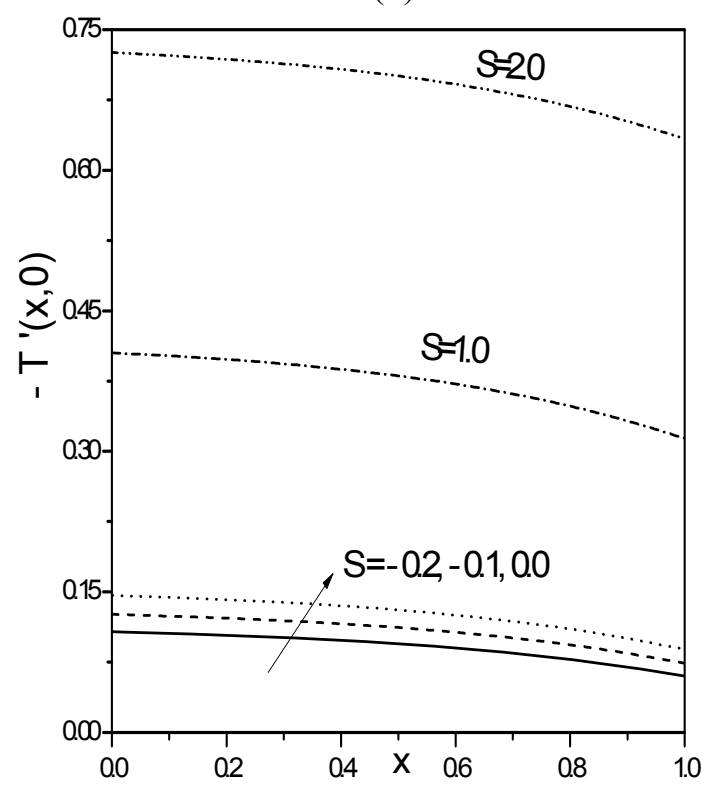

(d)

Fig.5. Effect of (a) Ec, (b) Bi, (c) $\lambda$ and (d) $S$ on $-T^{\prime}(x, 0)$. 
The behaviour of the mass transfer coefficient with $\tau$ against $x$ is portrayed in Fig.6a. It is seen from the figure that the rate of mass transfer is increasing with an increase in the value of $\tau$. Further, it is seen that for higher values of $\tau$ mass transfer from the sheet to the fluid is slightly decreasing gradually with $x$. The influence of the Biot number Bi on the mass transfer rate is presented in Fig.6b. From this figure we observe that the rate of mass transfer is increasing with an increase in $\mathrm{Bi}$ and non-similar variable $x$. The impact of slipperiness on the rate of mass transfer is shown in Fig.6c. From this, it is evident that the rate of mass transfer is reducing with hike in the value of $\lambda$ and $x$. Further, in the absence of the slip parameter there is a maximum mass transfer from the sheet to the fluid. But an opposite trend is observed on the rate of mass transfer when the slip parameter is replaced by the Biot number. Finally, the variation of the mass transfer coefficient for different values of suction/injection parameter $S$ is depicted in Fig.6d. This figure reveals that mass transfer from the sheet to the fluid is increasing with an increase in the value of suction and reducing with an enhancement in the value of injection. But, there is no effect of the non-similar variable $x$ on the mass transfer rate.

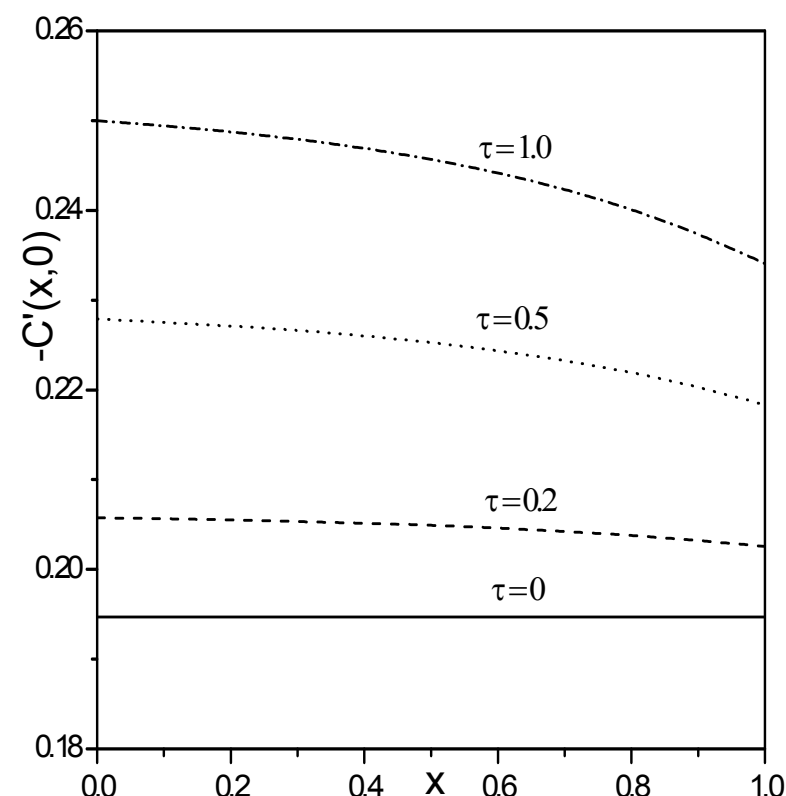

(a)

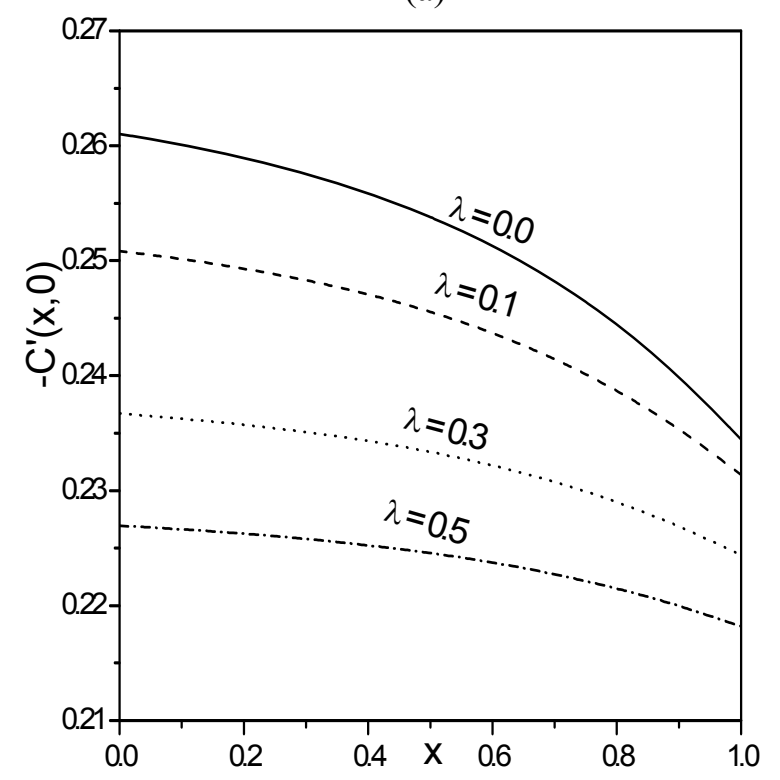

(c)

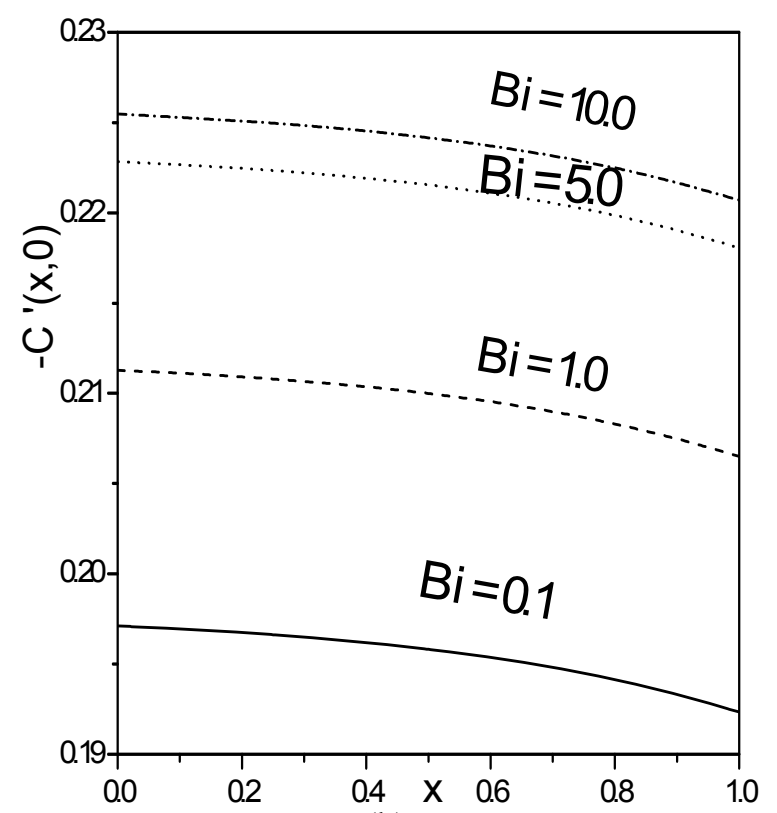

(b)

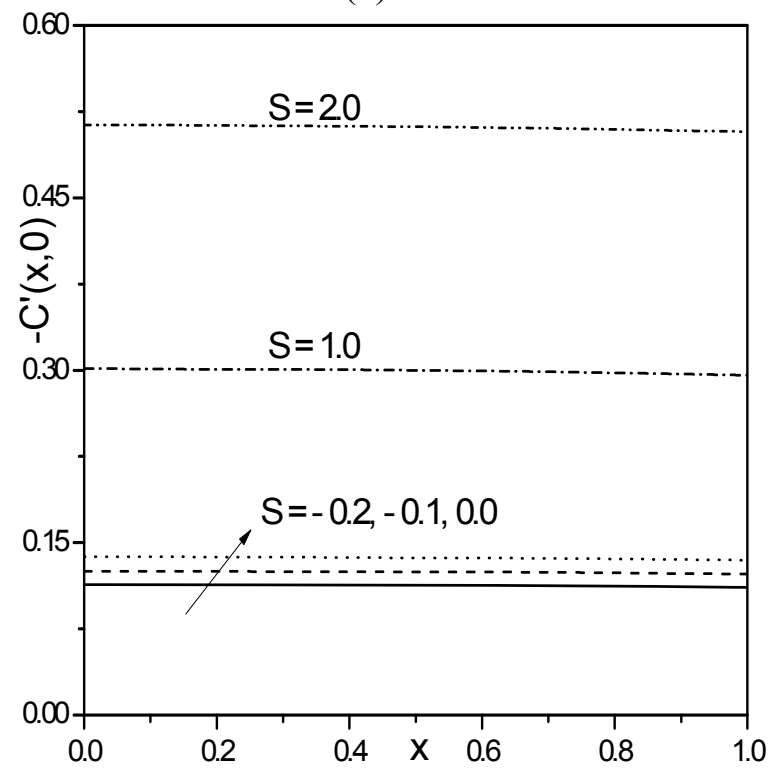

(d)

Fig.6. Effect of (a) $\tau$, (b) $\mathrm{Bi}$, (c) $\lambda$ and (d) $S$ on $-C^{\prime}(x, 0)$. 


\section{Conclusions}

The flow over a sheet stretching exponentially by considering the viscous dissipation and thermophoresis effects is investigated numerically by employing suction/injection, velocity slip and thermal convective boundary condition. A local similarity and non-similarity method along with successive linearisation and Chebyshev collocation method are used to solve the governing equations. The main findings are listed as follows.

- Velocity reduced as the value of suction/injection and slip parameters are increasing.

- Skin-friction increases with a rise in $\lambda$ and decreases with $S$.

- The temperature increased with a rise in the values of $\mathrm{Ec}, \mathrm{Bi}$ and $\lambda$. While decreased with enhancement in the value of $S$.

- Concentration of the fluid enhanced with increasing the value of $\lambda$ and reduced with an increase in the value of $\tau$, Bi and $S$.

- The rate of heat transfer rises with an enhancement in $\lambda, S$ and Bi and reduces with an increase in the value of the Eckert number.

- The rate of mass transfer from the sheet to the fluid increases with an increase in the values of $\tau, \mathrm{Bi}$ and $S$, but, decreases with increasing the value of $\lambda$.

\section{Nomenclature}

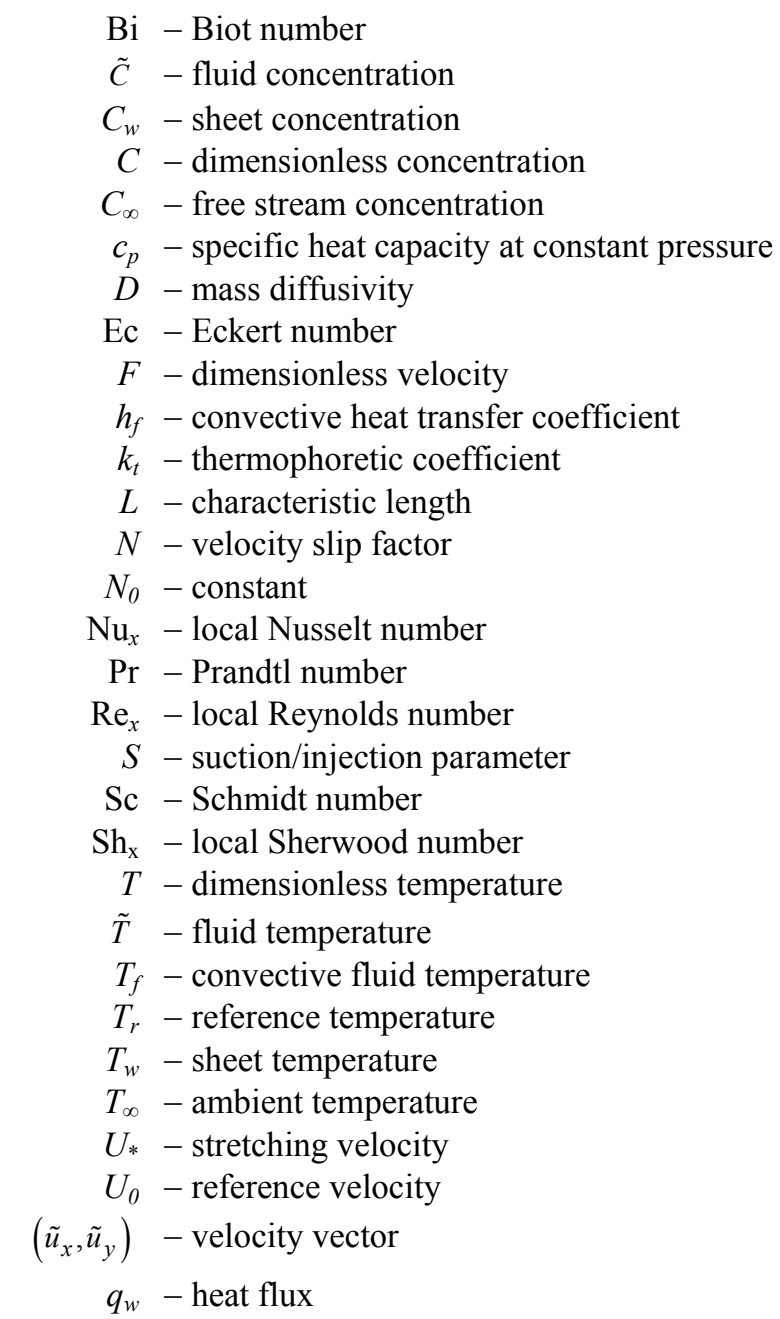


$q_{m}$ - mass flux

$V$ - variable wall mass transfer velocity

$V_{0}$ - constant

$V_{T}$ - thermophoretic velocity

$x$ - non-similar variable

$y$ - similarity variable

$\alpha-$ thermal diffusivity

$\kappa-$ thermal conductivity

$\lambda$ - slip parameter

$\mu-$ dynamic viscosity of the fluid

$\rho-$ density of the fluid

$\sigma$ - electrical conductivity

$\tau$ - thermophoretic parameter

$v$ - kinematic viscosity of the fluid

$\psi$ - stream function

\section{References}

[1] Sakiadis B.C. (1961): Boundary-layer equations for two-dimensional and axisymmetric flow. - A.I.Ch.E. Journal, vol.7, No.1, pp.26-28.

[2] Sakiadis B.C. (1961): The boundary layer on a continuous flat surface. - Vol.7, No.2, pp.221-225.

[3] Goldsmith P. and May F.G. (1966): Diffusiophoresis and thermophoresis in water vapour systems, Aerosol science. - Academic Press, pp.163-194.

[4] Uddin Md. J., Khan W.A. and Ismail A.I. Md. (2012): Scaling group transformation for MHD boundary layer slip flow of a nanofluid over a convectively heated stretching sheet with heat generation. - Mathematical Problems in Engineering, vol.2012.

[5] Shehzad S.A., Alsaedi A. and Hayat T. (2013): Influence of thermophoresis and Joule heaating on the radiative flow of Jeffrey fluid with mixed convection. - Brazilian Journal of Chemical Engineering, vol.30, No.4, pp.897908.

[6] Reddy M.G. (2014): Effects of thermophoresis, viscous dissipation and Joule heating on steady MHD flow over an inclined radiative isothermal permeable surface with variable thermal conductivity. - Journal of Applied Fluid Mechanics, vol.7, No.1, pp.51-61.

[7] Sandeep S. and Sulochana C. (2016): Dual solutions of radiative MHD nanofluid flow over an exponentially stretching sheet with heat generation/absorption. - Applied Nanoscience, vol.6, No.1, pp.131-139.

[8] Gebhart B. (1962): Effects of viscous dissipation in natural convection. - Journal of Fluid Mechanics, vol.14, No.2, pp.225-232.

[9] Wong S.W., Awang M.A.O., Ishak A. and Pop I. (2012): Boundary layer flow and heat transfer over an exponentially stretching/shrinking permeable sheet with viscous dissipation. - Journal of Aerospace Engineering, vol.27, No.1, pp.26-32.

[10] Das K. (2014): Influence of chemical reaction and viscous dissipation on MHD mixed convection flow. - Journal of Mechanical Science and Technology, vol.28, No.5, pp.1881-1885.

[11] Megahed A.M. (2015): Effect of slip velocity on Casson thin film flow and heat transfer due to unsteady stretching sheet in presence of variable heat flux and viscous dissipation. - Appl. Math. Mech..

[12] Adeniyan A. and Adigun J.A. (2016): Similarity Solution of hydromagnetic flow and heat transfer past an exponentially stretching permeable vertical sheet with viscous dissipation, Joulean and viscous heating effects. ANNALS of Faculty Engineering Hunedoara - International Journal of Engineering, pp.113-120.

[13] Naiver C.L.M. (1827): Sur les lois du mouvement des uides. - Memoires delAcademie Royale des Sciences, vol.6, pp.389-440. 
[14] Merkin J.H. (1994): Natural-convection boundary-layer flow on a vertical surface with Newtonian heating. International Journal of Heat and Fluid Flow, vol.15, No.5, pp.392-398.

[15] Gideon O.T. and Abah S.O. (2012): Plane stagnation double-diffusive MHD convective flow with convective boundary condition in a porous media. - American Journal of Computational Mathematics, vol.2, pp.223-227.

[16] Mustafaa M., Hayat T. and Obaidat S. (2013): Boundary layer flow of a nanofluid over an exponentially stretching sheet with convective boundary conditions. - International Journal of Numerical Methods for Heat and Fluid Flow, vol.23, No.6, pp.945-959.

[17] Hayat T., Saeed Y., Alsaedi A. and Asad S. (2015): Effects of convective heat and mass transfer in flow of PowellEyring fluid past an exponentially stretching sheet. - PloS one, vol.10, No.9, e0133831.

[18] Rahman M., Rosca A.V. and Pop I. (2015): Boundary layer flow of a nanofluid past a permeable exponentially shrinking surface with convective boundary condition using Buongiorno's model. - International Journal of Numerical Methods for Heat and Fluid Flow, vol.25, No.2, pp.299-319.

[19] Mabood F., Khan W.A. and Ismail A.I. Md. (2015): Approximate analytical solution of stagnation point flow and heat transfer over an exponential stretching sheet with convective boundary condition. - Heat Transfer-Asian Research, vol.44, No.4, pp.293-304.

[20] Khan J.A., Mustafa M., Hayat T. and Alsaedi A. (2015): Numerical study on three-dimensional flow of nanofluid past a convectively heated exponentially stretching sheet. - Canadian Journal of Physics, vol.93, No.10, pp.11311137.

[21] Srinivasacharya D. and Jagadeeshwar P. (2017): Slip viscous flow over an exponentially stretching porous sheet with thermal convective boundary conditions. - International Journal of Applied and Computational Mathematics, vol 3, No.4, pp.3525-3537.

[22] Talbot L., Cheng R.K., Schefer R.W. and Willis D.R. (1980): Thermophoresis of particles in a heated boundary layer. - Journal of Fluid Mechanics, vol.101, No.4, pp.737-758.

[23] Mills A.F., Xu Hang and Ayazi F. (1984): The effect of wall suction and thermophoresis on aerosol-particle deposition from a laminar boundary layer on a flat plate. - International Journal of Heat and Mass Transfer, vol.27, No.7, pp.1110-1113.

[24] Tsai R. (1999): A simple approach for evaluating the effect of wall suction and thermophoresis on aerosol particle deposition from a laminar flow over a flat plate. - International Communications in Heat and Mass Transfer, vol.26, No.2, pp.249-257.

[25] Sparrow E.M. and Yu H.S. (1971): Local non-similarity thermal boundary-layer solutions. - Transactions of the ASME

[26] Minkowycz W.J. and Sparrow E.M. (1974): Local nonsimilar solutions for natural convection on a vertical cylinder. Transactions of the ASME, pp.178-183.

[27] Srinivasacharya D. and Vijay Kumar P. (2015): Effect of thermal radiation and stratification on natural convection over an inclined wavy surface in a nanofluid saturated porous medium. - International Journal of Mining, Metallurgy and Mechanical Engineering, vol.3, No.1.

[28] Awad F.G., Sibanda P., Motsa S.S. and Makinde O.D. (2011): Convection from an inverted cone in a porous medium with cross-diffusion effects. - Computers and Mathematics with Applications, vol.61, pp.1431-1441.

[29] Canuto C., Hussaini M.Y., Quarteroni A. and Zang T.A. (2007): Spectral Methods-Fundamentals in Single Domains. - Journal of Applied Mathematics and Mechanics, vol.87, No.1.

Received: September 9, 2017

Revised: July 24, 2018 